\title{
Reflets
}

Revue ontaroise d'intervention sociale et communautaire

\section{La pratique en milieu francophone : réflexions de deux travailleuses sociales}

\section{Micheline Geoffrion-Lombardi et Angèle Bouchard}

Volume 1, numéro 1, printemps 1995

Des pratiques à notre image : défis et ressources

URI : https://id.erudit.org/iderudit/026060ar

DOI : https://doi.org/10.7202/026060ar

Aller au sommaire du numéro

Éditeur(s)

Reflets : Revue ontaroise d'intervention sociale et communautaire

ISSN

1203-4576 (imprimé)

1712-8498 (numérique)

Découvrir la revue

Citer cet article

Geoffrion-Lombardi, M. \& Bouchard, A. (1995). La pratique en milieu

francophone : réflexions de deux travailleuses sociales. Reflets, 1(1), 175-181.

https://doi.org/10.7202/026060ar

Tous droits réservés (C) Reflets : Revue ontaroise d'intervention sociale et communautaire, 1995
Ce document est protégé par la loi sur le droit d'auteur. L'utilisation des services d'Érudit (y compris la reproduction) est assujettie à sa politique d'utilisation que vous pouvez consulter en ligne.

https://apropos.erudit.org/fr/usagers/politique-dutilisation/ 


\section{La pratique en milieu francophone: réflexions de deux travailleuses sociales}

Micheline Geoffrion-Lombardi, M.s.s.

Travailleuse sociale, Centre psycho-social pour enfants et familles d'Ottawa-Carleton

avec la collaboration de

Angèle Bouchard, M.s.s.

Travailleuse sociale, Centre psycho-social pour enfants et familles d'Ottawa-Carleton

La pertinence d'offrir des services dans le contexte d'organismes francophones, plutôt que bilingues, à la population francoontarienne, est un sujet qui a été maintes fois débattu, mais qui demeure toujours d'actualité. Nous avons voulu saisir l'occasion de présenter le point de vue de deux travailleuses sociales oeuvrant au sein d'un centre de santé mentale exclusivement francophone, soit le Centre psycho-social pour enfants et familles d' OttawaCarleton.

Nous présenterons d'abord un bref historique de notre lieu de travail, pour ensuite souligner les avantages pour les thérapeutes et pour la clientèle de travailler et d'être servie dans sa langue maternelle, et aborder une notion que nous jugeons importante, soit celle de la culture. Notre réflexion se nourrit de nos rencontres avec la clientèle ainsi que de recherches et d'articles qui traitent de l' importance attribuée à la langue dans le respect des besoins des personnes auprès de qui l'on intervient. 
Reflets

\section{Aperçu historique}

Le Centre psycho-social pour enfants et familles, d'abord connu sous l'appellation de Centre régional pour enfants de l'hôpital Royal, a connu le jour à la fin des années soixante-dix.

À la suite des conclusions du Rapport Dubois (1976) qui démontraient la non-disponibilité des services de santé en langue française dans la province de l'Ontario, et plus spécifiquement dans les services de santé mentale au sein de la Municipalité régionale d'Ottawa-Carleton (MROC), le Conseil régional de santé d'Ottawa-Carleton se lie au Conseil de planification sociale afin de mettre sur pied un comité d'étude sur les services en français dans la région. Un sous-comité sur les services de psychiatrie pour enfants fut mis sur pied à l'Hôpital Royal d'Ottawa peu de temps après (1977).

Le rapport intérimaire de ce sous-comité souligne que, comme $17,5 \%$ des familles dans la région sont francophones, les services psychiatriques devraient être offerts en français dans la même proportion. Le Ministère des services sociaux et communautaires (MSCCO) débloque des fonds et en alloue une partie au développement d'une équipe multidisciplinaire en psychiatrie pour enfants au sein même de l'hôpital Royal. C'est en 1979 que cette équipe, composée d'un psychiatre, d'un psychologue, de deux travailleuses sociales, de deux psycho-éducateurs et de quelques spécialistes consultants, est mise sur pied.

En février 1982, le service est décentralisé pour être relocalisé dans la communauté francophone deVanier. Quelques mois plus tard, en novembre 1982, évoquant des restrictions budgétaires, l'hôpital Royal décide de dissoudre l'équipe francophone et d'intégrer ses membres au sein d'autres départements de l'hôpital. Réagissant à cette décision, la communauté francophone obtient le support du MSCCO pour la création d'un organisme francophone autonome qui offrira des services en santé mentale aux enfants et à leurs familles. Le Centre psycho-social fut créé un an plus tard (les documents d'incorporation furent émis en 1983). 
Les services sont alors offerts par quatre personnes qui, pour se joindre au Centre, avaient démissionné de l'équipe multidisciplinaire de l'Hôpital Royal.

Depuis, le Centre psycho-social pour enfants et familles d'Ottawa-Carleton ne cesse de croître. On note que le nombre de demandes de services a plus que doublé depuis 1983 alors que la population francophone n'a connu, elle, qu'une croissance d'environ 2,5\% depuis 1971.

Afin de mieux répondre aux besoins de sa clientèle, le Centre poursuit sa croissance et met sur pied de nouveaux services. Ainsi, en 1987, s'ouvre une garderie, Les petites frimousses. À cela s'ajoutent, en 1992, un Centre de jour pour les enfants de 4 à 6 ans (programme scolaire et thérapeutique au sein d'une école communautaire), en 1993, un service d'évaluation psycho-légale, et enfin, en septembre 1994, en partenariat avec le Conseil des écoles publiques d'Ottawa-Carleton, un programme éducatif pour parents/adolescents avec service de garde poupons-bambins. En juillet 1995, les services francophones présentement offerts par le Centre d'étude pour enfants seront intégrés au Centre psychosocial. Avec ce transfert, des services de jour pourront être offerts à 36 enfants âgés entre 4 et 11 ans et un programme résidentiel pour huit enfants de 6 à 11 ans viendra compléter la gamme de services offerts par le Centre.

\section{Les avantages du travail au sein d'un organisme francophone}

Nous sommes conscientes des nombreux avantages, en tant que thérapeutes francophones, d'occuper un emploi dans le seul centre francophone de santé mentale pour enfants d'Ottawa-Carleton.

Dans la plupart des autres agences, souvent désignées bilingues, bien que les cliniciens puissent offrir aux clients francophones des services directs dans leur langue maternelle, ils doivent le plus 
Reflets

souvent apprendre à fonctionner en anglais tant dans les contacts personnels avec leurs collègues que lors des réunions cliniques ou administratives. À cela s'ajoutent les divers travaux de rédaction qui doivent être préparés en anglais. Pour les intervenants ayant connu la frustration d'avoir à traduire et rédiger en anglais le compte rendu d'une intervention qui s' est déroulée en français, en tentant le plus possible de demeurer fidèle au contenu et au contexte, les avantages du travail dans un milieu entièrement francophone sont rapidement mis en lumière.

Ces avantages se vivent à plusieurs niveaux. En plus de faciliter la communication avec les clients, les entrevues en français permettent de mieux comprendre et définir leurs besoins. Pour nous, rédiger en français signifie plus de justesse, une économie de temps et d'énergie.

Au niveau de la communication verbale, c'est-à-dire la façon d'utiliser les mots, d'organiser les structures de phrases, de laisser place aux expressions locales et aux diverses sonorités des accents (Thomas, 1987), seule l'utilisation assidue d'une langue permet d'en saisir la richesse. Il est donc très important de bien maitriser le français pour bien saisir les diverses significations qui se cachent derrière les mots et expressions ordinaires.

Au niveau de la communication non-verbale, cette compréhension nous apparait tout aussi essentielle afin de pouvoir interpréter les éléments présents, qui sont en plus des gestes:

"...la façon de parler, de garder le silence, de regarder... Il s'agit aussi de pouvoir décoder les éléments du timbre de voix, de reconnaître la tristesse, la déception, la joie, l'excitation, etc., au-delà des mots.» (Thomas, 1987:5-7)

De plus, lors des réunions cliniques, pouvoir s'exprimer en français offre aux thérapeutes la possibilité de mieux traduire le vécu des clients sans perdre l'essence de leurs propos et de leurs émotions. Le fait d'échanger avec des collègues qui partagent une même langue favorise aussi le maintien d'une fierté de la langue et d'une identité linguistique souvent affaiblies et ébranlées dans un con- 
texte anglophone où l'on peut être soumis à des messages plus ou moins voilés sur l'importance du français en Ontario.

Il ne faudrait pas minimiser l'importance d'un accueil qui se traduit de diverses façons, que ce soit par les annonces, les diverses brochures ou affiches produites par le Centre, par le «bonjour» de la réceptionniste au téléphone ou lors de l'accueil; tous ces éléments contribuent à favoriser l'établissement $\mathrm{d}$ un premier contact qui soit positif et engageant.

À la notion de langue s'ajoute celle de la culture. Selon le sociologue Guy Rocher, dont on retrouve un court extrait dans le rapport de synthèse de Noël Thomas (1987), il faut:

"... être conscient des «façons de penser, de sentir et d'agir» du client et donc, par extension, être capable de composer avec ses façons de rêver, de solutionner ses problèmes, de planifier son avenir. Il faut donc être conscient des coutumes, des idées, des croyances, des traditions et des habitudes de la clientèle. Il faut en particulier être sensible, pour emprunter à l'analyse transactionnelle, aux injonctions parentales, culturelles et religieuses qui dictent le fonctionnement de l'être depuis sa tendre enfance et qui prennent racines dans la culture. Il faut donc comprendre tout le contexte psychique d'un individu et tout le contexte social de son milieu.»

Nous constatons que certains des avantages décrits précédemment favorisent non seulement les thérapeutes mais également la clientèle. En effet, en s'exprimant dans sa langue maternelle, chacun(e) peut mieux traduire ses émotions dans un vocabulaire qui fait référence au contexte, aux valeurs et à la culture qu'il véhicule. Le client se sent valorisé d'être francophone et rejoint dans son être intime parce qu'il est mieux compris. Nous avons pu observer qu'être servie en français favorisait, chez notre clientèle, une meilleure compréhension du processus et du fonctionnement du Centre, et contribuait à une réduction de son niveau d'anxiété. Toutefois, il faut reconnaître certaines limites auxquelles notre 
Reflets

Centre doit faire face. Quoiqu'il ait le mandat de desservir la région d'Ottawa-Carleton, le Centre demeure une organisation aux ressources modestes, et ses points de service sont limités. Le territoire d'Ottawa-Carleton est vaste et la population francophone, bien que concentrée dans certains secteurs, se retrouve un peu partout dans la région. Pour certains, le trajet jusqu'au centreville d'Ottawa ou de Vanier peut s'avérer long et, par conséquent, poser des difficultés de tout ordre. De plus, bien que l'on accepte les familles dans lesquelles l'un(e) des conjoints est unilingue anglophone, celles-ci ne peuvent bénéficier des services d'intervention de groupes qui sont offerts uniquement en français.

\section{Conclusion}

Dans l'ensemble, il apparaît assez clairement que, pour les francophones de notre région, avoir accès à des services en français signifie être respectés non seulement dans leur langue, mais dans leur culture et dans leur identité.

Nous croyons qu'à notre mandat d'offrir des services de qualité en français s'ajoute celui de promouvoir notre culture en vue de "...faciliter l'intégration du client dans sa communauté» (Thomas, 1987) et cela en brisant son isolement.

Le contexte socio-historique de la population francophone de l'Ontario continue d'évoluer. Les francophones de la région se sont, pendant de nombreuses années, trouvés sous l'égide d'une Église catholique forte qui se voyait chargée, non seulement de la mission de maintenir la foi, mais aussi celle de favoriser le développement de la culture et du fait français en plus de fournir les services sociaux et éducationnels. Ce rôle de pourvoyeur de services a été progressivement pris en main par l'État. Ce gouvernement étant, comme on le sait, à majorité anglophone, il s'en est suivi que la collectivité francophone s'est trouvée confrontée à un défi accru relativement à la conservation de sa langue et sa culture. En tant qu'agence française de services en santé mentale auprès des 
enfants et de leurs familles, le Centre psycho-social supporte et encourage à sa façon, selon ce que conçoit Mario Sarda (1990), les parents et les enseignants qui s'efforcent de maintenir vivant le fait français en Ontario.

En terminant, nous ne pouvons qu'appuyer les propos de l'honorable Thomas L. Wells à savoir qu'une

"... collectivité franco-ontarienne vigoureuse et saine en Ontario ne peut que nous apporter une meilleure compréhension des racines profondes et de la vitalité des deux nations fondatrices au sein de la Confédération canadienne.»

\section{Bibliographie}

CENTRE PSYCHO-SOCIAL POUR ENFANTS ET FAMILLES D'OTTAWA-CARLETON (1986). Historique du centre psycho-social.

CONSEIL DE PLANIFICATION SOCIALE D'OTTAWA-CARLETON (1985). Les professionnels francophones dans les services de santé et les services sociaux en Ontario.

DUBOIS, Jacques (1976). Pas de problèmes: Rapport sur les services de santé de langue française, Toronto, Ministère de la Santé.

SARDA, Mario (1990). Beyond Bilingualism: Providing Mental Health Services to Franco-Ontarian Children, Children's Mental Health, vol. 3, no 3, pp.2-6.

THOMAS, Noël (1987). La contribution des membres de la communauté à la mise en oeuvre de la loi de 1986 sur les services en français et au développement de services sociaux et communautaires en français, dans Guindon, René (sous la direction de), Rendons-nous service..., rapport synthèse du Forum sur les services en français en Ontario, l'Association canadienne-française de l'Ontario, pp.5-7. 